\title{
Chaotic oscillation in an attractive Bose-Einstein condensate under an impulsive force
}

\author{
Paulsamy Muruganandam and Sadhan K. Adhikari \\ Instituto de Física Teórica, Universidade Estadual Paulista, 01.405-900 São Paulo, São Paulo, Brazil
}

(Received 6 November 2001; published 26 March 2002)

\begin{abstract}
For an attractive trapped Bose-Einstein condensate an imaginary three-body recombination loss term and an imaginary linear source term are usually included in the Gross-Pitaevskii (GP) equation for a proper account of dynamics. Under the action of an impulsive force, generated by suddenly changing the atomic interaction or the trapping potential, the solution of this complex GP equation for attractive interaction is found to lead to a very long-term chaotic oscillation.
\end{abstract}

DOI: 10.1103/PhysRevA.65.043608

PACS number(s): 03.75.Fi

Since the successful detection [1,2] of Bose-Einstein condensates (BEC) in dilute bosonic atoms employing magnetic trap at ultralow temperature, there has been great theoretical and experimental interest in condensates with attractive interaction [2]. For attractive interaction the condensate is stable for a maximum critical number $N_{\text {cr }}$ of atoms [2]. When the number of atoms increases beyond this critical number, due to interatomic attraction the condensate collapses emitting atoms until the number of atoms is reduced below $N_{\text {cr }}$ and a stable configuration is reached. With a supply of atoms from an external source the condensate can grow again and thus a series of collapses can take place, which was observed experimentally in the BEC of ${ }^{7} \mathrm{Li}$ with attractive interaction [2]. Theoretical analyses based on the mean-field Gross-Pitaevskii (GP) equation $[3,4]$ also confirm the collapse [4-6].

In a recent classic experiment by Donley et al. on the BEC of ${ }^{85} \mathrm{Rb}$, a sustained harmonic oscillation of an attractive condensate had been observed and measured when the scattering length of atomic interaction was suddenly changed [7]. It is possible to manipulate the scattering length by an external magnetic field via a Feshbach resonance $[8,9]$. The sudden change in scattering length or the harmonic-oscillator trapping potential constitute a general class of impulsive force in the GP equation that can be realized experimentally. We show that under the action of an impulsive force the solution of the GP equation for attractive interaction with a quintic loss term and a linear source term can exhibit sustained chaotic oscillation. The imaginary quintic term in the GP equation accounts for three-body recombination loss and the imaginary linear term accounts for growth due to an external source of atoms.

There have been several studies of chaotic dynamics in the nonlinear Schrödinger [10], Landau-Ginzburg [11], and the GP $[12,13]$ equation. But all these studies were motivated from a numerical or mathematical point of view without phenomenological consequence. The chaotic oscillation we study here may be observed in the laboratory by slightly manipulating the strength of the linear source term responsible for supplying atoms to the condensate. In the absence of any source term or for a weak source term the oscillation is periodic after the application of the impulsive force. With a small increase in the source term the motion turns out to be chaotic. Donley et al. has performed a classic experiment where they have been able to observe and measure the fre- quency of oscillation of a condensate after the application of an impulsive force generated by changing the scattering length to a negative value [7]. Such an experiment might be able to detect the transition from periodic to chaotic oscillation of a condensate under the action of an impulsive force.

We consider for our present study on the numerical solution $[14,15]$ of the GP equation [3] for a spherically symmetric harmonic trap. In the GP equation we include a quintic three-body nonlinear recombination term that accounts for the decay of the strongly attractive condensate and a linear source term.

The GP equation in this case in dimensionless units can be written as $[5,6]$

$$
\begin{aligned}
& {\left[-\frac{\partial^{2}}{\partial x^{2}}+\frac{x^{2}}{4}+2 \sqrt{2} n\left|\frac{\varphi(x, t)}{x}\right|^{2}-i \xi\left|\frac{\varphi(x, t)}{x}\right|^{4}+i \gamma-i \frac{\partial}{\partial t}\right]} \\
& \times \varphi(x, t)=0,
\end{aligned}
$$

where $n=N a / l, \gamma$ is the coefficient of the linear source term, and $\xi$ is the coefficient of the quintic three-body recombination term. Here the distance $x$, time $t$, and the spherically symmetric wave function $\phi(x, t) \equiv \varphi(x, t) / x$ are expressed in units of $l / \sqrt{2}, \omega^{-1}$, and $\left(\sqrt{2} \pi l^{3}\right)^{-1 / 2}$, where $l=\sqrt{(\hbar / m \omega)}$ and $\omega$ is the frequency of the harmonic-oscillator trap, $m$ is the mass of a single atom, $N$ is the number of atoms in the condensate, and $a$ the scattering length of atomic interaction. The normalization condition for the wave function is

$$
\int_{0}^{\infty} d x|\varphi(x, t)|^{2}=1 .
$$

We solve Eq. (1) above by the Crank-Nicholson time propagation [14] after discretization with $\gamma \neq 0$ and $\xi \neq 0$ starting from the known harmonic-oscillator solution for $n=\xi=\gamma$ $=0$. The $x$ discretization was performed with space step 0.1 up to a maximum $x$ of 40 . The time step was taken to be 0.01 . The nonlinearity constant $n$ was increased by steps of 0.0001 until the desired value is reached. Once the final nonlinearity is reached the solution is then stabilized by iterating 100000 times, which corresponds to an interval of time $t$ $=1000$. This removes any transient behavior in the solution that is important for a study of chaos. Then the wave function is prepared for the simulation of chaos after the application of the impulsive force. The oscillation of the system 
after the application of the impulsive force is best studied by considering the time evolution of the root-mean-square (rms) radius $X$ and its time derivative $\dot{X}$.

For attractive interaction, a stable condensate can be formed for the minimum value -0.575 for $n$ [4-6]. We consider the following three numerical simulations for the study of chaos: (i) On a preformed attractive condensate with $n=$ -0.4 we suddenly double the harmonic-oscillator term from $x^{2} / 4$ to $x^{2} / 2$ and study the resultant oscillation of the system for different $\gamma$. On a preformed repulsive condensate with $n=+0.4$, we suddenly change the sign of the scattering length $a$ to (ii) $-a$ and also to (iii) $-2 a$ and study the resultant oscillation of the system for different $\gamma$. In all simulations we take $\xi=0.0004$. In cases (i) and (ii) the final value of $n(=-0.4>-0.575)$ permits stable condensate and in case (iii) final value of $n(=-0.8<-0.575)$ does not permit a stable solution of Eq. (1). However, the experiment conducted at JILA [7] has shown that in case (iii) the number of particles cannot decay immediately below the stability limit and there could be sustained oscillation of the system with a value of $n$ less than -0.575 . We find that depending on the value of the source term $\gamma$ there could be pronounced chaos in all three cases above.

Once the wave function is prepared for the study of chaos as described above we inflict the change corresponding to case (i), first, with $\gamma=0$. Only periodic oscillation of rms radius $X$ and its derivative $\dot{X}$ is observed. With a slight increase in $\gamma$ to 0.0002 , chaotic oscillation is obtained. The change from periodic to chaotic motion is best illustrated by plotting $\dot{X}$ vs $X$. For a periodic motion a closed loop appears in the phase-space plot of $\dot{X}$ vs $X$, whereas for a chaotic motion a strange attractor appears in the phase-space plot. This is shown in Figs. 1 (a) and 1(b) for $\gamma=0.0001$ (periodic) and 0.0002 (chaotic), respectively, for an interval of simulation time $t=200$ after inflicting the impulsive force. With the increase of $\gamma$, the periodic motion of Fig. 1(a) changes to chaotic motion in Fig. 1(b). The present time is expressed in units of $\omega^{-1}$. In a typical experimental situation the harmonic-oscillator trapping frequency $\nu \sim 50 \mathrm{~s}^{-1}$, and hence $\omega^{-1}=(2 \pi \nu)^{-1} \sim 0.005 \mathrm{~s}$. Thus the simulation time of $t=200$ corresponds to $1 \mathrm{~s}$ that is inside the experimental observation period of a typical setup [7]. We continued the simulation till $t=20000$ and the robust chaotic attractor seems to stay forever, although it moves slowly to a smaller value of $X$ with time. This is exhibited in Fig. 1(c), where we plot $\dot{X}$ vs $X$ for $0<t<20000$ that corresponds to an interval of $100 \mathrm{~s}$.

Next we perform a similar simulation as in Fig. 1 above for the case (ii) where the sign of the scattering length is suddenly changed from positive to negative exploiting a Feshbach resonance as shown in the experiment conducted at JILA [7]. In simulation this corresponds to changing $n$ from 0.4 to -0.4 (repulsive to attractive). The final $n$ ( $=-0.4>$ -0.575 ) allows for a stable attractive condensate to be formed. Again for $\gamma=0$ and 0.0001 periodic motions are obtained that change to chaotic motion as $\gamma$ is increased. In Fig. 2 we plot $\dot{X}$ vs $X$ for $\gamma=0.0001$ and $\gamma=0.0002$ as in

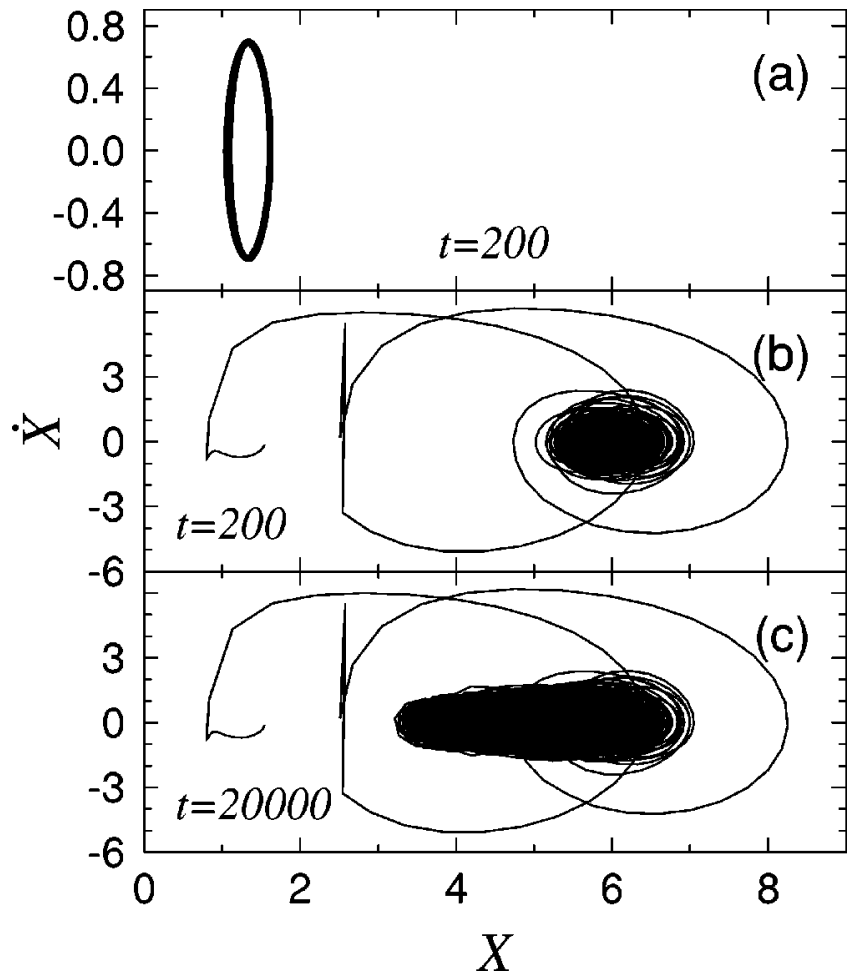

FIG. $1 . \dot{X}$ vs $X$ in case (i) when the harmonic-oscillator potential is suddenly doubled in Eq. (1) for $\xi=0.0004, n=-0.4$ and (a) $\gamma$ $=0.0001, t=200$, (b) $\gamma=0.0002, t=200$, and (c) $\gamma=0.0002, t$ $=20000$.

Fig. 1. In Fig. 2(a) we show the periodic motion for $\gamma$ $=0.0001$ for time $t$ up to 200. Fully chaotic motion is obtained for $\gamma=0.0002$. The chaotic motion for the initial interval of time 200 is shown in Fig. 2(b) and that for an interval of 20000 units of time is shown in Fig. 2(c). The robust chaotic attractor has started to develop in Fig. 2(b) and its long-term evolution is shown in Fig. 2(c).

Finally, we consider the case (iii) above. In this case the nonlinear term $n$ has been suddenly changed from 0.4 to -0.8 (repulsive to attractive). However, in this case the final $n(=-0.8<-0.575)$ does not allow a stable condensate to be formed. Such a strongly attractive condensate has been created and observed in the laboratory in the experiment conducted at JILA [7]. In this case the condensate is unstable and due to interatomic attraction it starts to shrink in size or collapse after the sudden change in the nonlinear term. Consequently, as the central density of the condensate increases, it starts to emit particle through small explosions and tries to attain a more stable configuration with a smaller number of particles [7]. The condensate exhibits oscillation during this process of collapse and explosion but it may need a large amount of time before attaining the critical size with $N_{\text {cr }}$ atoms. No periodic oscillation was observed in this case even for $\gamma=0$. In Fig. 3(a) we plot $\dot{X}$ vs $X$ for the first 200 units of time for $\gamma=0$, which shows a chaotic attractor, which continues to exist for any positive nonzero $\gamma$. In Fig. 3(b) we plot the same for $\gamma=0.0002$, which shows the strange attractor for the first 200 units of time. In Fig. 3(c) we exhibit the long-term behavior of this attractor for 20000 units of time. 


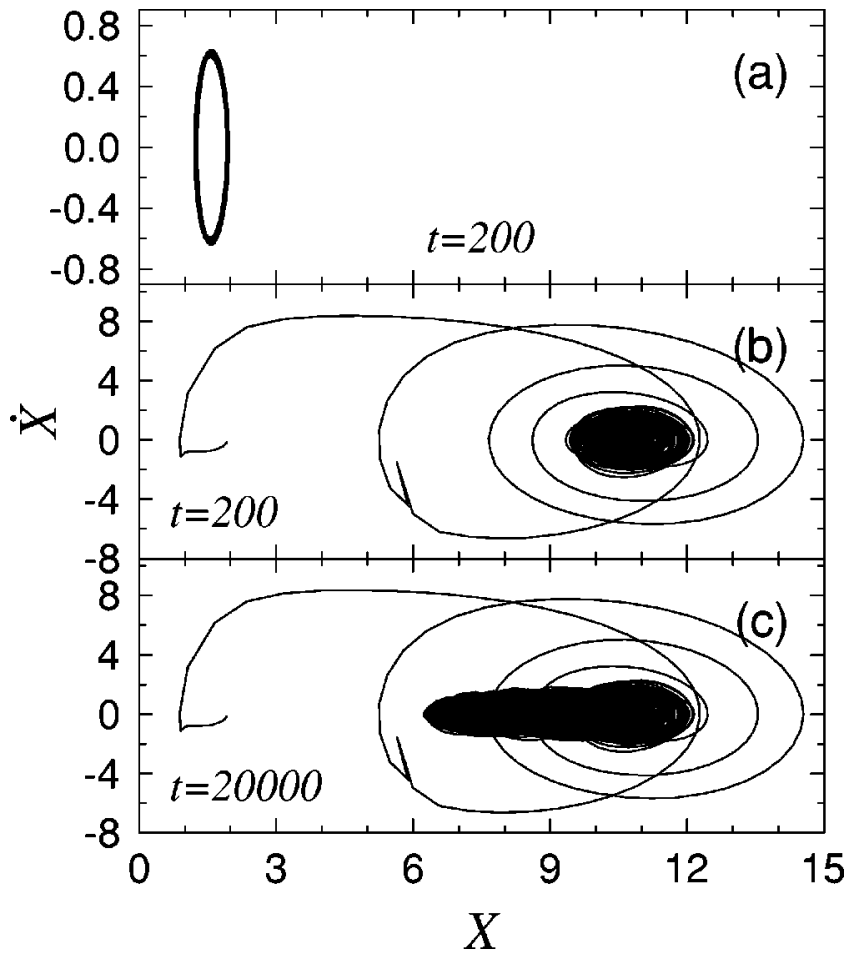

FIG. 2. $\dot{X}$ vs $X$ in case (ii) when the sign of the scattering length $a$ was suddenly made negative from positive in Eq. (1) for $\xi$ $=0.0004, n=0.4$ and (a) $\gamma=0.0001, t=200$, (b) $\gamma=0.0002, t$ $=200$, and (c) $\gamma=0.0002, t=20000$.

In the long term the attractor is more spread in this case compared to Figs. 1(c) and 2(c). In case (iii) in the long term the chaotic attractor moves to large $X$ whereas in cases (i) and (ii) it moves to smaller $X$.

The presence of chaos is characterized by finding the Lyapunov exponents [16]. Since the quantities $X$ and $\dot{X}$ are not direct solutions of the nonlinear GP equation (1) and are essentially the expectation values, we use time-series analysis to calculate the Lyapunov exponents [16]. In all the cases, we use the velocity-variable $(\dot{X})$ data collected with the time interval $\delta t=0.1$. The reason to choose $\dot{X}$ is that it oscillates with time only around the steady average zero value, whereas the time average of $X$ is not stationary and changes with time. From one set of data for $\dot{X}(t)$ we construct other independent set(s) by allowing a time lag $\tau$. The number of such independent sets constitute the embedding dimension $m$ of the data sets for $\dot{X}(t)$ to be analyzed. The optimal time lag is found from the estimate of mutual information that shows the independent nature of the reconstructed data sets [17]. After some experimentation we find that $m=3$ leads to reasonable values for all the Lyapunov exponents. All the calculations of the Lyapunov exponents are performed with $m$ $=3$ using the algorithm by Sano and Sawada [18].

The calculation leads to three exponents in each of the cases (i), (ii), and (iii) with $\gamma=0.0002$ shown in Figs. 1(c), 2(c), and 3(c), respectively. In case (i), the time lag $\tau$ in the calculation of Lyapunov exponents was found to be 0.6 and in cases (ii) and (iii) it was found to be 0.9. In Fig. 4 we plot the largest Lyapunov exponent versus the time used for the

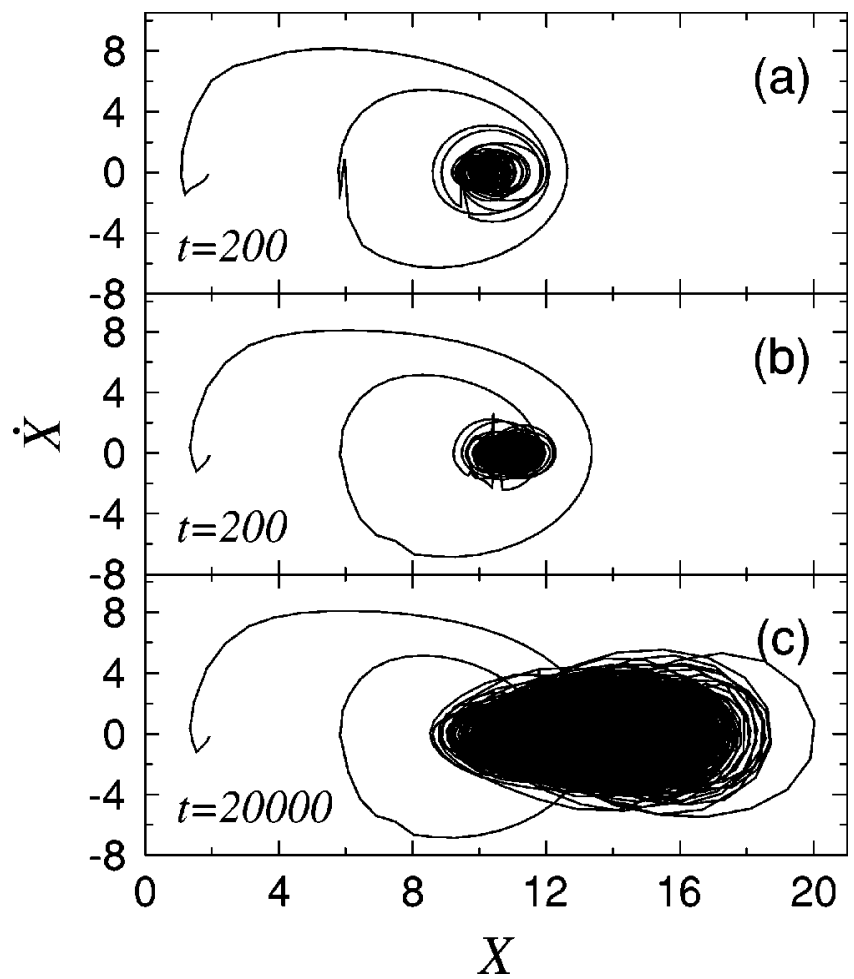

FIG. 3. $\dot{X}$ vs $X$ in case (iii) when the scattering length $a$ was suddenly changed to $-2 a$ in Eq. (1) for $\xi=0.0004, n=0.4$ and (a) $\gamma=0, t=200$, (b) $\gamma=0.0002, t=200$, and (c) $\gamma=0.0002, t$ $=20000$.

calculation in the three cases above. In all three cases the largest exponent is found to lead to a convergent positive value at large finite time that shows the existence of chaos in all the cases. We also found that the oscillation in Fig. 3(a) corresponding to case (iii) with $\gamma=0$ is also chaotic.

There has been confirmation of chaos in the nonlinear Schrödinger equation [10] and in the Landau-Ginzburg equation [11] with similar nonlinear terms as in the GP equation, although the details of the two equations are different. Also, there has been theoretical prediction of chaos in a coupled set of GP equations [12]. The numerical study of chaos in a

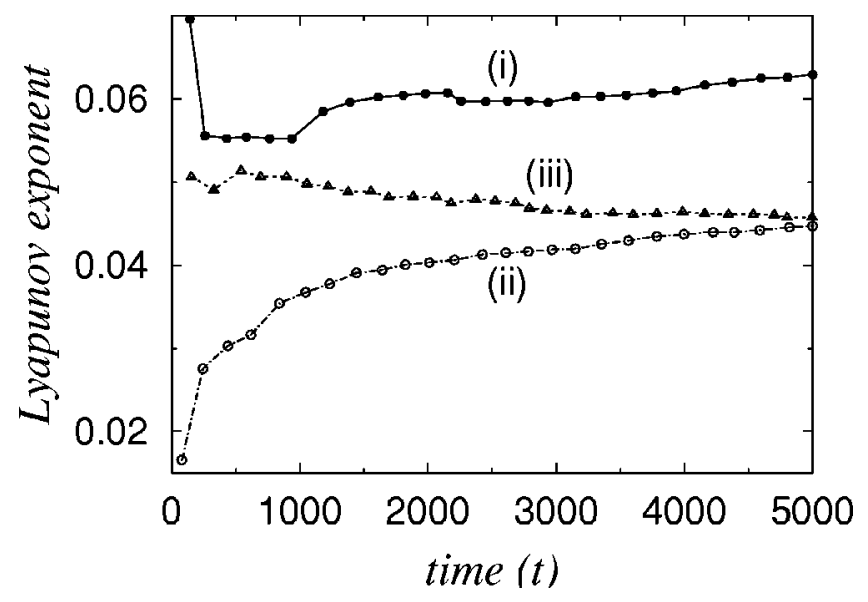

FIG. 4. Lyapunov exponents for the chaotic attractors of Figs. 1(c), 2(c), and 3(c) labeled (i), (ii), and (iii), respectively. 
collapsing Bose-condensed gas in Ref. [13] is worth mentioning. In that work chaos was confirmed in the normal time evolution of the GP equation (1) without any external impulsive force. The chaotic attractor in that study was found to stay only for a short interval of time $(\sim 500)$, where in the present study with impulsive forces the chaotic oscillations are found to appear immediately after the application of the impulsive force and stay for more than 20000 units of time $t$. This makes the analysis of the chaos via Lyapunov exponent more reliable.

The appearance of chaos in nonlinear dynamics is of interest from a theoretical point of view. Here we have demonstrated the chaotic oscillation in an attractive BEC under an impulsive force using the mean-field GP equation. The use of the GP equation in this paper is justified as this equation produces a faithful representation of the BEC for both repulsive and attractive interactions $[5,6]$. We also performed simulation when the condensate is repulsive after the application of the impulsive force. No chaotic oscillation was detected in that case. From this it seems that the final-state interatomic attraction plays an important role in the generation of this chaotic dynamics.

In summary, from a numerical simulation based on the solution of the Gross-Pitaevskii equation (1) for attractive interaction with an absorptive quintic three-body recombination term $(\xi)$ and a linear source term $\gamma$, we find that sustained chaotic oscillation can result in a BEC under the action of an impulsive force generated by suddenly changing the interatomic scattering length or the harmonic-oscillator trapping potential.

The work was supported in part by the Conselho Nacional de Desenvolvimento Científico e Tecnológico and Fundação de Amparo à Pesquisa do Estado de São Paulo of Brazil.
[1] J.R. Ensher, D.S. Jin, M.R. Matthews, C.E. Wieman, and E.A. Cornell, Phys. Rev. Lett. 77, 4984 (1996); K.B. Dadic, M.O. Mewes, M.R. Andrews, N.J. van Druten, D.S. Durfee, D.M. Kurn, and W. Ketterle, ibid. 75, 3969 (1995); D.G. Fried, T.C. Killian, L. Willmann, D. Landhuis, S.C. Moss, D. Kleppner, and T.J. Greytak, ibid. 81, 3811 (1998); F. Pereira Dos Santos, J. Léonard, J. Wang, C.J. Barrelet, F. Perales, E. Rasel, C.S. Unnikrishnan, M. Leduc, and C. Cohen-Tannoudji, ibid. 86, 3459 (2001).

[2] J.M. Gerton, D. Strekalov, I. Prodan, and R.G. Hulet, Nature (London) 408, 692 (2001); C.C. Bradley, C.A. Sackett, J.J. Tollett, and R.G. Hulet, Phys. Rev. Lett. 75, 1687 (1995); C.C. Bradley, C.A. Sackett, and R.G. Hulet, ibid. 78, 985 (1997).

[3] E.P. Gross, Nuovo Cimento 20, 454 (1961); L.P. Pitaevskii, Zh. Éksp. Teor. Fiz. 40, 646 (1961) [Sov. Phys. JETP 13, 451 (1961)].

[4] F. Dalfovo, S. Giorgini, L.P. Pitaevskii, and S. Stringari, Rev. Mod. Phys. 71, 463 (1999).

[5] H. Saito and M. Ueda, Phys. Rev. Lett. 86, 1406 (2001); R.A. Duine and H.T.C. Stoof, ibid. 86, 2204 (2001); A. Eleftheriou and K. Huang, Phys. Rev. A 61, 043601 (2000).

[6] Y. Kagan, E.L. Surkov, and G.V. Shlyapnikov, Phys. Rev. Lett. 79, 2604 (1997); Y. Kagan, A.E. Muryshev, and G.V. Shlyapnikov, ibid. 81, 933 (1998); M. Ueda and A.J. Leggett, ibid. 80, 1576 (1998); A. Gammal, T. Frederico, L. Tomio, and Ph. Chomaz, Phys. Rev. A 61, 051602 (2000); M. Ueda and K. Huang, ibid. 61, 043601 (1999); R.J. Dodd, M. Edwards, C.J. Williams, C.W. Clark, M.J. Holland, P.A. Ruprecht, and K. Burnett, ibid. 54, 661 (1996).

[7] E.A. Donley, N.R. Claussen, S.L. Cornish, J.L. Roberts, E.A. Cornell, and C.E. Wieman, Nature (London) 412, 295 (2001).
[8] S. Inouye, M.R. Andrews, J. Stenger, H.J. Miesner, D.M. Stamper-Kurn, and W. Ketterle, Nature (London) 392, 151 (1998); E. Timmermans, P. Tommasini, M. Hussein, and A. Kerman, Phys. Rep. 315, 199 (1999).

[9] J.L. Roberts, N.R. Claussen, S.L. Cornish, E.A. Donley, E.A. Cornell, and C.E. Wieman, Phys. Rev. Lett. 86, 4211 (2001); S.L. Cornish, N.R. Claussen, J.L. Roberts, E.A. Cornell, and C.E. Wieman, ibid. 85, 1795 (2000).

[10] M.J. Ablowitz, C. Schober, and B.M. Herbst, Phys. Rev. Lett. 71, 2683 (1993).

[11] R. Braun and F. Feudel, Phys. Rev. E 53, 6562 (1996); R.J. Deissler and K. Kaneko, Phys. Lett. A 119, 397 (1987); H.R. Brand and R.J. Deissler, Phys. Rev. E 58, R4064 (1998).

[12] F.K. Abdullaev and R.A. Kraenkel, Phys. Rev. A 62, 023613 (2000); P. Coullet and N. Vandenberghe, Phys. Rev. E 64, 025202 (2001).

[13] V.S. Filho, A. Gammal, T. Frederico, and L. Tomio, Phys. Rev. A 62, 033605 (2000).

[14] S.K. Adhikari, Phys. Rev. E 62, 2937 (2000); 63, 056704 (2001); 65, 016703 (2002); S.K. Adhikari, Phys. Rev. A 63, 043611 (2001); Phys. Lett. A 281, 265 (2001); 265, 91 (2000); J. Phys. B 34, 4231 (2001).

[15] M. Holland and J. Cooper, Phys. Rev. A 53, R1954 (1996); A. Gammal, T. Frederico, and L. Tomio, ibid. 64, 055602 (2001); F. Dalfovo and S. Stringari, ibid. 53, 2477 (1996); P.A. Ruprecht, M.J. Holland, K. Burnett, and M. Edwards, ibid. 51, 4704 (1995).

[16] R. Hegger, H. Kantz, and T. Schreiber, Chaos 9, 413 (1999).

[17] A.M. Fraser and H.L. Swinney, Phys. Rev. A 33, 1134 (1986).

[18] M. Sano and Y. Sawada, Phys. Rev. Lett. 55, 1082 (1985). 